\title{
Factors associated with high-risk low-level viremia leading to virologic failure: 16-year retrospective study of a Chinese antiretroviral therapy cohort
}

Tong Zhang ${ }^{1,2,3,4,5+}{ }^{+}$, Haibo Ding ${ }^{1,2,3,4,5+}$, Minghui An ${ }^{1,2,3,4,5}$, Xiaonan Wang ${ }^{1,2,3,4,5}$, Wen Tian ${ }^{1,2,3,4,5}$, Bin Zhao $1,2,3,4,5$ and Xiaoxu Han $1,2,3,4,5^{*}$

\begin{abstract}
Background: Low level viremia (LLV) often occurs during antiretroviral therapy (ART) against HIV-1. However, whether LLV increases the risk of virologic failure (VF) is controversial because of the non-uniform definitions of LLV and VF.

Methods: A long-term first line regimen ART cohort from 2002 to 2018 from Shenyang, northeast China, was retrospectively studied. All participants were followed up every 3 to 6 months to evaluate the treatment effect. The high-risk LLV subgroups leading to VF (with strict standards) were explored with Cox proportional hazards model and linear mixed-effect model. The association factors of high-risk LLV were further explored using multivariate logistic regression analyses.

Results: A total of 2155 HIV-1 infected participants were included; of these, 38.7\% showed LLV. Both high level LLV (HLLV) and any other level LLV coupled with high level blip (HLB) showed higher risk of VF (hazards ratios, HR $R_{\text {HLLV }}=$ 5.93, and $H R_{H L B}=2.84, p<0.05$ respectively). Moreover, HR increased with prolonged duration of LLV. Independent factors associated with high-risk LLV included the zenith baseline viral load $(\mathrm{VL})$ above 6 log copies $/ \mathrm{ml}(\mathrm{aOR}=3.49$, $p=0.002$ ), nadir baseline CD4 + T cell counts below 200 cells $/ \mathrm{mm}^{3}$ ( $\left.\mathrm{aOR}=1.78, p=0.011\right)$, Manchu $(\mathrm{aOR}=2.03$, $p=0.003)$, ART over 60 months (aOR $=1.81, p=0.004), A Z T+3 T C+N V P(a O R=2.26, p<0.001)$ or DDI-based regimen $(\mathrm{aOR}=9.96, p=0.002)$, and subtype $\mathrm{B}^{\prime}$ infection $(\mathrm{aOR}=8.22, p=0.001)$.
\end{abstract}

Conclusions: In case of VF with strict standards, high-risk LLV leading to VF includes VL above 400 copies/ml, occurring at least once. Serious laboratory indicators or advanced stage of infection, long term ART and subtype $B^{\prime}$ infection might also predict the occurrence of high-risk LLV.

Keywords: HIV-1, Viral load assay, Low-level viremia, Virologic failure, Long-term antiretroviral therapy, First-line regimen, Lower-income countries

\footnotetext{
* Correspondence: hanxiaoxu@cmu.edu.cn

${ }^{\dagger}$ Tong Zhang and Haibo Ding contributed equally to this work.

${ }^{1}$ NHC Key Laboratory of AIDS Immunology (China Medical University),

Department of Laboratory Medicine, The First Affiliated Hospital of China

Medical University, No 155, Nanjing North Street, Heping District, Shenyang

110001, Liaoning Province, China

${ }^{2}$ National Clinical Research Center for Laboratory Medicine, The First Affiliated

Hospital of China Medical University, Shenyang 110001, China

Full list of author information is available at the end of the article
}

(c) The Author(s). 2020 Open Access This article is distributed under the terms of the Creative Commons Attribution 4.0 International License (http://creativecommons.org/licenses/by/4.0/), which permits unrestricted use, distribution, and reproduction in any medium, provided you give appropriate credit to the original author(s) and the source, provide a link to the Creative Commons license, and indicate if changes were made. The Creative Commons Public Domain Dedication waiver (http://creativecommons.org/publicdomain/zero/1.0/) applies to the data made available in this article, unless otherwise stated. 


\section{Background}

Human immunodeficiency virus type 1 (HIV-1) rapidly replicates mainly in CD4 $+\mathrm{T}$ cells and is released into the blood. Antiretroviral therapy (ART) can limit virus replication to less than 50 copies/ml and effectively prolong the life of HIV-1 infected patients [1]. However, in approximately $10-30 \%$ of the patients, depending on the definition, standard ART cannot control viral replication completely $[2,3]$. The plasma of such patients shows low level of viral replication, which is designated as low level viremia (LLV) [4]. Among these cases, the occasionally detected viremia is designated as viral blip (Blip) [5], whereas the continuously detected viremia is designated as persistent low level viremia (pLLV) [6]. The standards of LLV vary greatly with different guidelines. In resource-limited countries or regions $[2,7,8]$, LLV is defined as a viral load (VL) between 50 and 1000 copies/ $\mathrm{ml}$, according to the World Health Organization (WHO) guidelines [9]. In United States and some other resource-rich countries [10-12], LLV is defined as a VL between 50 and 200 copies $/ \mathrm{ml}$ according to the Department of Health and Human Services (DHHS) guidelines (USA, 2016) [13]. In Europe and most resource-rich settings [14, 15], LLV is defined as a VL between 20 and 50 copies $/ \mathrm{ml}$, according to the European acquired immune deficiency syndrome (AIDS) Clinical Society (EACS) guidelines [16]. Although LLV frequently occurs during treatment, none of the guidelines suggest changing the treatment regimen or intensifying treatment in case of LLV.

The impact of LLV on clinical prognosis is still controversial. An earlier national observational cohort study from USA found that an LLV above 400 copies $/ \mathrm{ml}$ could not predict AIDS or death [17]. However, multiple recent cohort studies worldwide demonstrated that the level of LLV is a determinant of prognosis; high level of LLV (> 200 copies/ml) occurring only once might increase the risk of virologic failure (VF) [7, 14, 18]. Some studies from developed countries found that persistent LLV or frequently occurring LLV of 50-200 copies/ml also increased the risk of VF $[6,19,20]$. However, a recent multicenter study from South Africa found that LLV below 200 copies/ml, whether continuous or not, contributed to increased VF risk [2]. Moreover, several recent studies found that even very low levels of pLLV between 20 and 50 copies $/ \mathrm{ml}$ could predict an increased risk of viral failure $[14,21]$. Most of the above studies varied in their standards of LLV or VF definitions, and in some studies, the definition of VF was not stringent. For example, a recent multicenter cohort study reported that approximately $20 \% \mathrm{VF}$ patients, according to the standard of WHO guideline, could spontaneously control viral load to below 1000 copies/ml during the follow up time without any intervention; however, these cases did not strictly belong to the VF category [2]. Moreover, no study has evaluated the impact of LLV on the risk of VF considering both level and duration of LLV.

Although most studies suggest that LLV contributes to VF, the potential risk factors of LLV have not been sufficiently investigated. Several HIV cohort studies from developed countries where subtype B was predominant found that the high zenith baseline VL and low nadir baseline $\mathrm{CD} 4+\mathrm{T}$ cell counts were associated with the risk of $\operatorname{LLV}[3,22-25]$. However, the evidence from non-subtype $\mathrm{B}$ infected cases and resource-limited regions are still lacking.

The aims of the current study, which used clinical data from a long-term first line regimen ART cohort in China, were to determine the characteristics of high-risk LLV associated with VF, and to elucidate the factors contributing to this kind of LLV, by considering both the level and the duration of LLV. The results of this study might provide new evidence for the occurrence and contribution of risk factors of LLV, and thereby help to optimize ART treatment in resource-limited setting.

\section{Methods \\ Study population}

This retrospective study was based on an open HIV-1 infection treatment cohort in a big comprehensive hospital in Shenyang, northeastern China between 2002 and 2018. All subjects received first-line ART regimen according to Chinese guidelines for diagnosis and treatment of HIV/AIDS [26]. This included two nucleoside reverse transcriptase inhibitors (NRTIs) and a non- nucleoside reverse transcriptase inhibitor (NNRTI) that are widely used globally [27-31]. All participants were followed up every 3 to 6 months for evaluation of $\mathrm{VL}, \mathrm{CD} 4+\mathrm{T}$ cell counts, and other routine clinical parameters. The socio-demographic information included gender, age at HIV diagnosis ( $<50$ years), marital status, education, ethnicity, steady income, and transmission route. The clinical data, including duration on ART (months), ART regimen, and laboratory test results including $\mathrm{VL}, \mathrm{CD} 4+\mathrm{T}$ cell counts, and HIV genotype, were collected from clinical follow-up case records.

The inclusion criteria for this study were as follows: (1) participants received first-line ART regimen in China (2 NRTIs and 1 NNRTI); (2) VL had been controlled to less than $50 \mathrm{copies} / \mathrm{ml}$ after 6 months treatment; (3) participants were followed-up for at least 12 months. Patients were excluded from this study if their VLs were incomplete. This study was approved by the ethics committee of the First Affiliated Hospital of China Medical University. In addition, informed consents were obtained from all participants. 


\section{Definitions of LLV and VF}

In this study, we used the definition of LLV based on the WHO guideline [9]; LLV was defined as viremia between 50 and 1000 copies/ml during ART, and was classified into three groups: high level LLV of 400-1000 copies/ml (HLLV), medium level LLV of 200-400 copies/ml (MLLV), and low level LLV of 50-200 copies/ml (LLLV). Any level LLV coupled with a high level blip of above 1000 copies/ml was defined as high level blip (HLB). The definition of VF was modified on the basis of the WHO standard to two or more consecutive VL of above 1000 copies $/ \mathrm{ml}$ and viral replication that could not be controlled spontaneously without changing therapeutic regimens.

\section{Statistical analyses}

The CD4 lymphocyte count and the results of viral load were included as quantitative variables. The K-S normal test method was used to evaluate the normality of variables. The comparisons were made between LLV categories by log-rank tests. Chi-squared test was used for comparisons among other variables. The incidence of VF was calculated by the total number of VF occurrence/total follow-up time * $100 \%[32,33]$. The impacts of LLV on VF were evaluated by the Cox proportional hazards model and the linear mixed-effect model (shown with hazard ratio (HR) and estimate with their 95\%CI), in which VL were analyzed as time-varying covariates [7]. The characteristics of high risk LLV that increased the risk of subsequent VF were determined. Multiple factors, consisting of socio-demographic information, transmission route, clinical data, and laboratory test results, were used to identify potential factors of high-risk LLV by multivariate logistic regression, with correction for sex and age [34]. Survival analysis was performed to calculate the association between all LLV categories and VF. Kaplan-Meier curves were created for the entire study population according to the level and duration of LLV. In creating the survival curves, time to event (i.e. VF) was estimated from the date of ART initiation to the date of VF; in absence of VF, we used the date of last clinic visit as the follow-up time. All statistical analyses were conducted using Statistical Package for the Social Sciences software (SPSS 22.0) and a $p$-value of less than 0.05 was considered statistically significant.

\section{Results}

Characteristics of participants in this study

In total, 2155 participants were selected from a longterm, first-line ART regimen, local Chinese cohort (Fig. 1). The patients in this study were followed up to 8348 person-years in total, and with the median follow up time of 3.4 years (IQR: 2.3-5.0). The majority of the participants were male $(92.9 \%, 2001 / 2155)$, with a

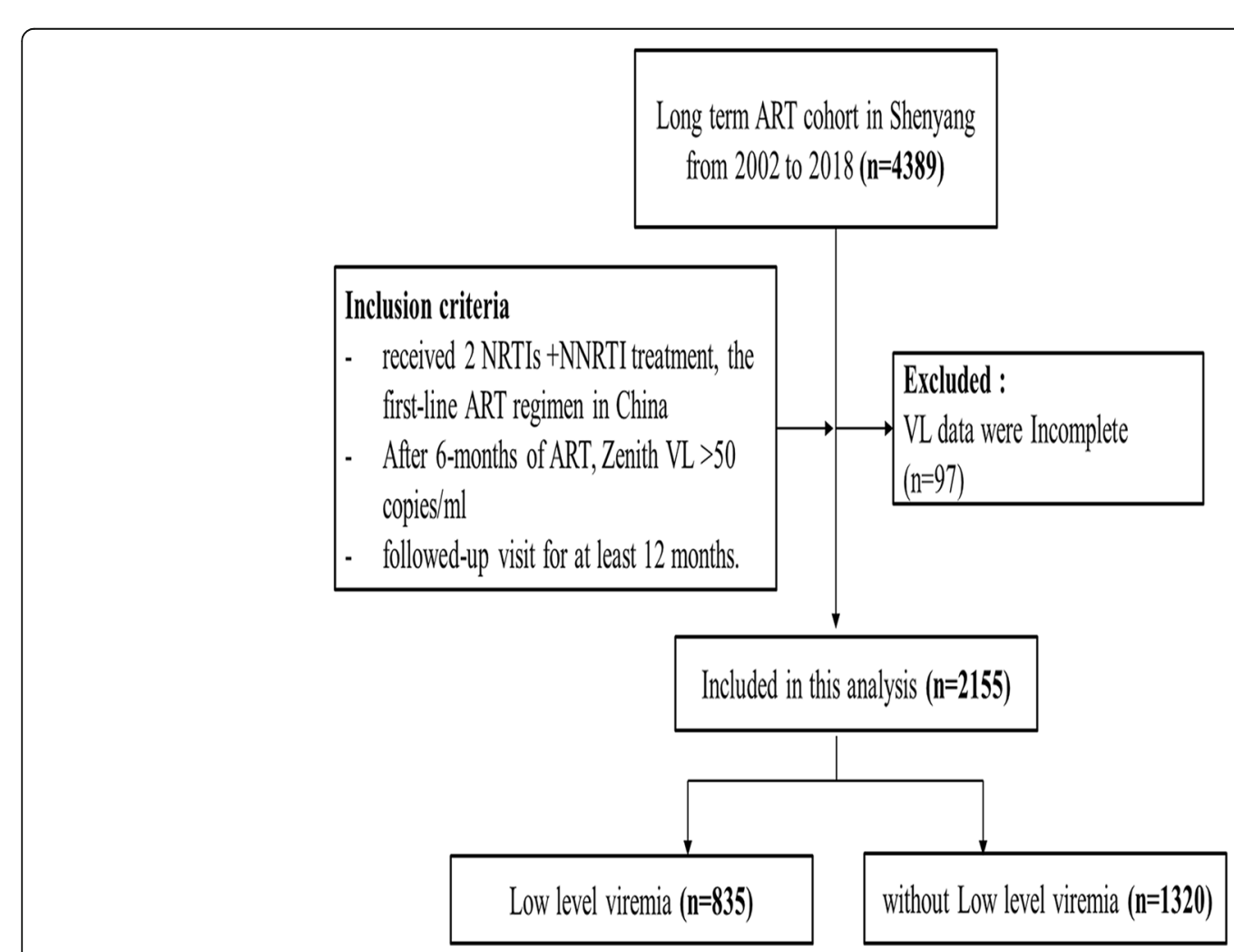

Fig. 1 Flowchart showing selection of the participants 
median age at HIV diagnosis of 37 years old (IQR: 3149). They mainly comprised of the Han nationality (85.4\%). Further, 79.2\% (1706/2155) of the participants were infected with HIV-1 through homosexual transmission, $65.1 \%$ (1403/2155) were infected with CRF01_AE HIV-1, and $68.5 \%(1477 / 2155)$ of participants used $\mathrm{TDF}+3 \mathrm{TC}+\mathrm{EFV}$ as the ART regimen. Most participants $(50.9 \%, 1096 / 2155)$ showed the zenith baseline VL of 4-5 (log copies/ml) and 37.4\% (805/2155) of participants displayed the nadir baseline $\mathrm{CD} 4+\mathrm{T}$ cell counts of 200-350 cells $/ \mathrm{mm}^{3}$ (Additional file 1: Table S1).

In this study, $38.7 \%(835 / 2155)$ of participants experienced at least one episode of LLV. In terms of the LLV level, participants belonging to LLLV, MLLV, HLLV, and HBL groups accounted for 52.1\% (435/835), $18.0 \%$ (150/835), $14.3 \%$ (119/835), and $15.7 \%(131 / 835)$, respectively. In terms of the duration of LLV, participants with Blip, LLV lasting for 3-6 months, 6-12 months, and above 12 months accounted for $72.1 \%(602 / 835), 11.4 \% \quad(95 / 835)$,
$11.4 \% \quad(95 / 835)$, and $5.1 \% \quad(43 / 835)$, respectively (Additional file 1: Table S1).

\section{High risk LLV contributes to VF}

The VF rate increased along with increasing level of LLV (50-200 copies/ml: 1.6\%, 200-400 copies/ml: 4.7\%, 400-1000 copies/ml: $13.4 \%$, Fig. $2 \mathrm{a}$ ) and the duration of viremia (Blip: 3.3\%, 3-6 months: 7.4\%, 6-12 months: $6.3 \%$, > 12 months: $11.6 \%$, Fig. $2 \mathrm{~b}$ ). On the basis of the estimated Kaplan-Meier curves, there were significant differences in the risk of VF $(p<0.01)$ between LLV level and duration categories. Compared with the control group (incidence rate (IR): 0.52/100 person years), those with $>400$ copies/ml (IR: $2.97 / 100$ person years, $p<0.01$ ) and LLV duration of $>12$ months (IR: 3.68/100 person years, $p<0.01$ ) had the least favorable KaplanMeier curves and higher risk of VF (Fig. 2c, d). The Cox proportional hazards model showed that only HLLV (HR: 5.93, 95\%CI: 3.13-11.23, $p<0.01$ ) and HBL (HR: 2.84, 95\%CI: $1.27-6.34, p=0.011$ ) were associated with
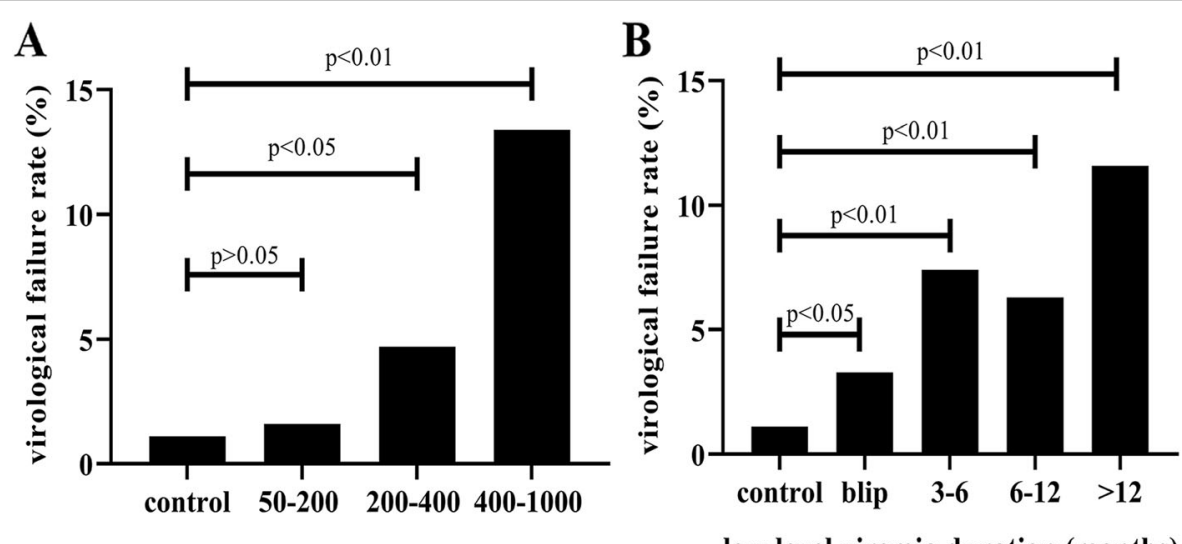

low level viremia range (copies/ml)

low level viremia duration (months)
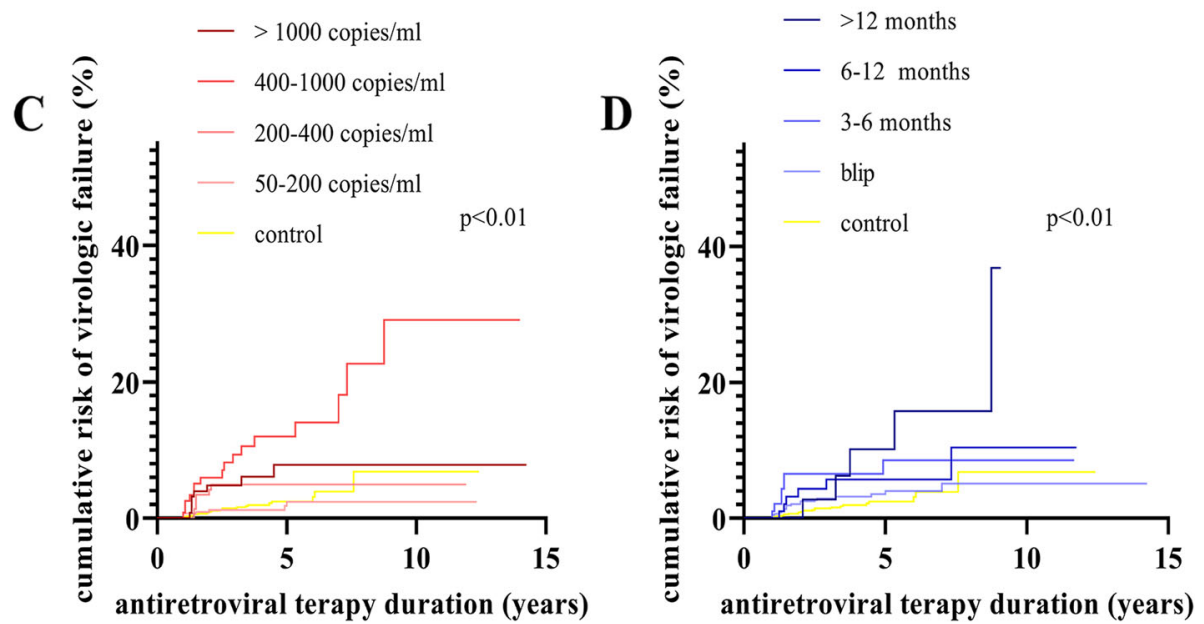

Fig. 2 Incidence of virologic failure according to the level and the duration of low level viremia (LLV). Incidence of virologic failure according to the (a) level of LLV, and (b) duration of LLV. Kaplan-Meier estimate for virologic failure according to the (c) level of LLV, and (d) duration of LLV 
significantly increased risk of subsequent VF compared with the control group (all VL below 50 copies $/ \mathrm{ml}$ ).

Based on LLV duration analyses, we found that the risk of VF increased in the HLLV group for any duration; moreover, the HRs increased with prolonged duration (Blip: $\mathrm{HR}=4.41,95 \% \mathrm{CI}: 1.96-9.92, p<0.01$; 3-6 months: $\mathrm{HR}=6.59, \quad 95 \% \mathrm{CI}: 1.94-22.34, p<0.01 ; 6-12$ months: $\mathrm{HR}=5.19,95 \% \mathrm{CI}: 1.22-22.18, p<0.05$; and $>12$ months: $\mathrm{HR}=9.63$, 95\%CI: 2.82-32.95, $p<0.01)$. In addition, any level LLV (>3 months) coupled with HBL also showed increased risk of VF (3-6 months: $\mathrm{HR}=6.20,95 \% \mathrm{CI}$ : $1.46-26.27, p<0.05 ; 6-12$ months: $\mathrm{HR}=9.74,95 \% \mathrm{CI}$ : 2.82-33.66, $\quad p<0.01$; $>12$ months: $\mathrm{HR}=7.52, \quad 95 \% \mathrm{CI}$ : 1.01-55.84, $p<0.05)$. In contrast, no significant increase of VF was observed among MLLV and LLLV groups, even in those that lasted for $>12$ months $(200-400$ copies $/ \mathrm{ml}$ : $\mathrm{HR}=3.43$, 95\%CI: $0.46-25.54, p>0.05 ; 50-200$ copies $/ \mathrm{ml}$ : $\mathrm{HR}=1.02$, 95\%CI: 1.01-1.03, $p>0.05)$.

In this study, VL was determined at 3-6 months interval and acted as a time-varying covariate. We further used the linear mixed-effect model to validate the contribution of LLV on VF [35, 36]. Our results supported the contribution of HLLV and HLB to VF, but the MLLV group showed weak association with increased risk of VF (estimated 0.03, 95\%CI: $0.00-0.06, p=0.045$ ). LLLV showed no increase of VF in both models (Table 1).

\section{Factors associated with high risk LLV}

We further assessed the socio-demographic information, transmission route, clinical data, and laboratory test results to explore the factors associated with high risk LLV. Using chi-square tests, we found that the different groups of zenith baseline VL, nadir CD4 + T cell counts, and other demographic information, clinical data, and laboratory tests had varied frequencies of high risk LLV (Table 2). Multiple logistic regression analysis further supported that participants with higher zenith baseline VL ( $>6 \log$ copies/ml vs. $<4$ log copies $/ \mathrm{ml}: \mathrm{aOR}=3.49$, 95\%CI: $1.55-7.83, p<0.01)$ and lower nadir baseline $\mathrm{CD} 4+\mathrm{T}$ cell counts $\left(<200\right.$ cells $/ \mathrm{mm}^{3}$ vs. $>350$ cells/ $\left.\mathrm{mm}^{3}: \mathrm{aOR}=1.78,95 \% \mathrm{CI}: 1.14-2.78, p<0.05\right)$ showed increased risk of high-risk LLV. Other associated factors included Manchu (aOR: 2.03, 95\%CI: 1.27-3.25,

Table 1 Association between LLV characteristics and virologic failure in the linear mixed-effect model and the Cox proportional hazards model $(N=2155)$

\begin{tabular}{|c|c|c|c|c|c|}
\hline \multirow[t]{2}{*}{ LLV group $^{a}$} & \multirow[t]{2}{*}{ Case Number } & \multicolumn{2}{|c|}{ Cox proportional hazards model } & \multicolumn{2}{|c|}{ Linear mixed-effect model } \\
\hline & & $\mathrm{HR}(95 \% \mathrm{Cl})$ & $p$-value & Estimate $(95 \% \mathrm{Cl})$ & $p$-value \\
\hline$<50$ (copies/ml) & 1320 & 1(reference) & & O(reference) & \\
\hline 50-200 (copies/ml) & 435 & $0.72(0.31-1.68)$ & 0.453 & $0.00(-0.02-0.02)$ & 0.819 \\
\hline blip $p^{b}$ & 318 & $0.68(0.26-1.80)$ & 0.438 & $0.00(-0.02-0.01)$ & 0.765 \\
\hline $3-6$ (months) $^{c}$ & 47 & $1.58(0.37-6.80)$ & 0.541 & $0.02(-0.01-0.06)$ & 0.213 \\
\hline 6-12 (months) ${ }^{d}$ & 53 & $1.02(1.01-1.03)$ & 0.975 & $-0.02(-0.05-0.02)$ & 0.325 \\
\hline$>12$ (months) $)^{e}$ & 17 & $1.02(1.01-1.03)$ & 0.986 & $-0.02(-0.08-0.04)$ & 0.572 \\
\hline 200-400 (copies/ml) & 150 & $1.90(0.81-4.43)$ & 0.139 & $0.03(0.00-0.06)$ & 0.045 \\
\hline blip ${ }^{b}$ & 108 & $1.91(0.72-5.06)$ & 0.196 & $0.03(0.00-0.06)$ & 0.051 \\
\hline $3-6$ (months) $^{c}$ & 14 & $1.02(1.01-1.03)$ & 0.979 & $-0.02(-0.10-0.06)$ & 0.637 \\
\hline $6-12$ (months) $^{d}$ & 17 & $2.12(0.28-15.99)$ & 0.468 & $0.04(-0.03-0.11)$ & 0.246 \\
\hline$>12$ (months) $^{e}$ & 11 & $3.43(0.46-25.54)$ & 0.229 & $0.07(-0.01-0.16)$ & 0.094 \\
\hline 400-1000 (copies/ml) & 119 & $5.93(3.13-11.23)$ & 0.000 & $0.12(0.09-0.15)$ & 0.000 \\
\hline blip $^{b}$ & 78 & $4.41(1.96-9.92)$ & 0.000 & $0.08(0.05-0.12)$ & 0.000 \\
\hline 3-6 (months) ${ }^{c}$ & 18 & $6.59(1.94-22.34)$ & 0.002 & $0.15(0.07-0.22)$ & 0.000 \\
\hline $6-12$ (months) ${ }^{d}$ & 14 & $5.19(1.22-22.18)$ & 0.026 & $0.12(0.04-0.21)$ & 0.004 \\
\hline$>12$ (months) $^{e}$ & 9 & $9.63(2.82-32.95)$ & 0.000 & $0.32(0.21-0.42)$ & 0.000 \\
\hline HLB > 1000 (copies/ml) & 131 & $2.84(1.27-6.34)$ & 0.011 & $0.04(0.01-0.07)$ & 0.005 \\
\hline blip ${ }^{b}$ & 98 & $0.97(0.23-4.09)$ & 0.961 & $0.00(-0.03-0.03)$ & 0.883 \\
\hline 3-6 (months) ${ }^{c}$ & 16 & $6.20(1.46-26.27)$ & 0.013 & $0.11(0.04-0.19)$ & 0.003 \\
\hline 6-12 (months) ${ }^{d}$ & 11 & $9.74(2.82-33.66)$ & 0.000 & $0.25(0.17-0.34)$ & 0.000 \\
\hline$>12$ (months) $^{\mathrm{e}}$ & 6 & $7.52(1.01-55.84)$ & 0.049 & $0.15(0.03-0.27)$ & 0.012 \\
\hline
\end{tabular}

a: LLV group is defined according to the Zenith VL during ART, subgroup is further divided according to the duration of LLV at any level between 50 and 1000 copies/ml. b: single viral blip, c: pLLV lasts 3 months, d: pLLV lasts 6 months, e: pLLV lasts more than 12 months. VL viral load, $H R$ hazard ratio, $C l$ confidence interval, HLB high level blip. The bold data in the table shows the subgroup with increased risk of the subsequent VF, which we defined as high-risk LLV 
Table 2 Factors associated with high risk LLV

\begin{tabular}{|c|c|c|c|c|c|c|c|}
\hline \multirow[t]{2}{*}{ Characteristics } & \multirow{2}{*}{$\begin{array}{l}\text { Case } \\
\text { Number }\end{array}$} & \multirow{2}{*}{$\begin{array}{l}\text { High risk LLV } \\
\text { case number (\%) }\end{array}$} & \multirow[t]{2}{*}{$p$-value } & \multicolumn{2}{|c|}{ Unadjusted analysis } & \multicolumn{2}{|l|}{ Adjusted analysis } \\
\hline & & & & $\mathrm{OR}(95 \% \mathrm{Cl})$ & $p$-value & $\mathrm{aOR}(95 \% \mathrm{Cl})$ & $p$-Value \\
\hline \multicolumn{8}{|l|}{ Gender } \\
\hline Male & 2001 & 142(7.10) & 0.957 & 1.00(reference) & & & \\
\hline Female & 125 & $8(6.40)$ & & $1.03(0.24-4.38)$ & 0.967 & & \\
\hline NA & 29 & $2(6.90)$ & & $0.92(0.19-4.60)$ & 0.922 & & \\
\hline \multicolumn{8}{|l|}{ Age at HIV diagnosis } \\
\hline$\leq 50$ (years) & 1618 & $110(6.80)$ & 0.607 & 1.00(reference) & & & \\
\hline$>50$ (years) & 500 & $40(8.00)$ & & $0.78(0.19-3.30)$ & 0.739 & & \\
\hline NA & 37 & $2(5.41)$ & & $1.19(0.82-1.74)$ & 0.361 & & \\
\hline \multicolumn{8}{|l|}{ Nation } \\
\hline Han & 1840 & $123(6.68)$ & 0.017 & 1.00(reference) & & 1.00(reference) & \\
\hline Manchu & 195 & $24(12.31)$ & & $1.96(1.23-3.12)$ & 0.005 & $2.03(1.27-3.25)$ & 0.003 \\
\hline Others & 82 & $3(3.66)$ & & $0.53(0.17-1.70)$ & 0.287 & $0.55(0.17-1.76)$ & 0.310 \\
\hline NA & 38 & $2(5.26)$ & & $0.78(0.19-3.26)$ & 0.729 & $0.60(0.10-3.64)$ & 0.579 \\
\hline \multicolumn{8}{|l|}{ Duration on ART } \\
\hline 12-36(months) & 954 & $57(5.97)$ & 0.004 & 1.00(reference) & & 1.00(reference) & \\
\hline 36-60(months) & 674 & $41(6.08)$ & & $1.02(0.67-1.54)$ & 0.928 & $1.02(0.67-1.54)$ & 0.925 \\
\hline$>60$ (months) & 527 & $54(10.25)$ & & $1.80(1.22-2.65)$ & 0.003 & $1.81(1.21-2.69)$ & 0.004 \\
\hline \multicolumn{8}{|l|}{ ART regimen } \\
\hline $\mathrm{TDF}+3 \mathrm{TC}+\mathrm{EFV}$ & 1477 & 84(5.69) & $<0.001$ & 1.00(reference) & & 1.00(reference) & \\
\hline $\mathrm{AZT}+3 \mathrm{TC}+\mathrm{EFV}$ & 276 & $20(7.25)$ & & $1.30(0.78-2.15)$ & 0.315 & $1.31(0.79-2.18)$ & 0.301 \\
\hline$A Z T+3 T C+N V P$ & 294 & $35(11.90)$ & & $2.24(1.48-3.40)$ & $<0.001$ & $2.26(1.48-3.45)$ & $<0.001$ \\
\hline $\mathrm{D} 4 \mathrm{~T}+3 \mathrm{TC}+\mathrm{EFV}$ & 41 & $5(12.20)$ & & $2.30(0.88-6.02)$ & 0.089 & $2.38(0.90-6.33)$ & 0.082 \\
\hline $\mathrm{D} 4 \mathrm{~T}+3 \mathrm{TC}+\mathrm{NVP}$ & 59 & $5(8.47)$ & & $1.54(0.60-3.94)$ & 0.372 & $1.56(0.61-4.04)$ & 0.355 \\
\hline $\mathrm{DDI}+\mathrm{a}$ NRTI +a NNRTI & 8 & $3(37.50)$ & & $9.95(2.34-42.34)$ & 0.002 & $9.96(2.33-42.49)$ & 0.002 \\
\hline \multicolumn{8}{|l|}{ Transmission route } \\
\hline Sexual & 1965 & 132(6.72) & 0.133 & 1.00(reference) & & 1.00(reference) & \\
\hline Others & 24 & $2(8.33)$ & & $1.26(0.29-5.43)$ & 0.754 & $1.31(0.30-5.67)$ & 0.715 \\
\hline NA & 166 & 18(10.84) & & $1.69(1.00-2.84)$ & 0.048 & $1.84(1.05-3.23)$ & 0.034 \\
\hline \multicolumn{8}{|l|}{ HIV-1 subtype } \\
\hline $\mathrm{B}$ & 130 & $7(5.38)$ & 0.001 & 1.00(reference) & & 1.00(reference) & \\
\hline $\mathrm{B}^{\prime}$ & 20 & $6(30.00)$ & & $7.53(2.22-25.57)$ & 0.001 & $8.22(2.34-28.92)$ & 0.001 \\
\hline CRF01_AE & 1403 & $99(7.06)$ & & $1.33(0.61-2.94)$ & 0.474 & $1.32(0.60-2.90)$ & 0.495 \\
\hline CRF07_BC & 213 & 19(8.92) & & $1.72(0.70-4.21)$ & 0.235 & $1.73(0.71-4.23)$ & 0.232 \\
\hline Others & 95 & $4(4.21)$ & & $0.77(0.22-2.72)$ & 0.687 & $0.77(0.22-2.73)$ & 0.690 \\
\hline $\mathrm{Na}$ & 294 & $17(5.78)$ & & $1.08(0.44-2.67)$ & 0.870 & $1.09(0.44-2.71)$ & 0.849 \\
\hline \multicolumn{8}{|l|}{ Zenith baseline VL } \\
\hline$<4\left(\log _{10}\right.$ copies/ml) & 535 & $30(5.61)$ & 0.006 & 1.00(reference) & & 1.00(reference) & \\
\hline $4-5\left(\log _{10}\right.$ copies/ml) & 1096 & $72(6.57)$ & & $1.18(0.76-1.84)$ & 0.452 & $1.18(0.76-1.83)$ & 0.469 \\
\hline 5-6 (log 10 copies/ml) & 472 & $41(8.69)$ & & $1.60(0.98-2.61)$ & 0.059 & $1.58(0.96-2.58)$ & 0.070 \\
\hline$>6\left(\log _{10}\right.$ copies/ml) & 52 & $9(17.31)$ & & $3.52(1.57-7.90)$ & 0.002 & $3.49(1.55-7.83)$ & 0.002 \\
\hline \multicolumn{8}{|c|}{ Nadir baseline CD4+ cell counts } \\
\hline$>350($ cells/ml) & 665 & $34(5.11)$ & 0.058 & 1.00(reference) & & 1.00(reference) & \\
\hline 200-350 (cells/mm³) & 805 & $57(7.08)$ & & $1.41(0.91-2.19)$ & 0.121 & $1.41(0.91-2.19)$ & 0.123 \\
\hline
\end{tabular}


Table 2 Factors associated with high risk LLV (Continued)

\begin{tabular}{|c|c|c|c|c|c|c|c|}
\hline \multirow[t]{2}{*}{ Characteristics } & \multirow{2}{*}{$\begin{array}{l}\text { Case } \\
\text { Number }\end{array}$} & \multirow{2}{*}{$\begin{array}{l}\text { High risk LLV } \\
\text { case number (\%) }\end{array}$} & \multirow[t]{2}{*}{$p$-value } & \multicolumn{2}{|c|}{ Unadjusted analysis } & \multicolumn{2}{|l|}{ Adjusted analysis } \\
\hline & & & & OR(95\%Cl) & $p$-value & $\mathrm{aOR}(95 \% \mathrm{Cl})$ & $p$-Value \\
\hline$<200\left(\right.$ cells $\left./ \mathrm{mm}^{3}\right)$ & 634 & $56(8.83)$ & & $1.80(1.16-2.79)$ & 0.009 & $1.78(1.14-2.78)$ & 0.011 \\
\hline NA & 51 & $5(9.80)$ & & $2.02(0.75-5.40)$ & 0.163 & $2.03(0.76-5.43)$ & 0.160 \\
\hline
\end{tabular}

Adjusted for gender and age

LLV low level viremia, participants with VL results range of $>50$ copies per $\mathrm{ml}$ but not defined as virologic failure, $O R$ odds ratio, $a O R$ adjusted odds ratio, Cl confidence interval, NA Not available, ART antiretroviral therapy, HIV human immunodeficiency virus, TDF Tenofovir, 3TC Lamivudine, EFV Efavirenz, AZT

Zidovudine, NVP Nevirapine, D4T Stavudine, DDI didanosine, NRTIs nucleotide analogue reverse transcriptase inhibitors, NNRTIs nonnucleotide Analogue Reverse Transcriptase Inhibitors

$p<0.01$ ), ART for over 60 months (aOR: $1.81,95 \% \mathrm{CI}$ : $1.21-2.69, p<0.01)$, receiving AZT $+3 \mathrm{TC}+\mathrm{NVP}$ regimen (aOR: $2.26,95 \% \mathrm{CI}: 1.48-3.45, p<0.01$ ) or $\mathrm{DDI}+$ NRTI+NNRTI regimen (aOR: 9.96, 95\%CI: 2.33-42.49, $p<0.01$ ) and subtype $\mathrm{B}^{\prime}$ infection (aOR: $8.22,95 \% \mathrm{CI}$ : $2.34-28.92, p<0.01)$.

\section{Discussion}

In this study, we observed LLV from $38.7 \%$ of participants in a local long-term ART cohort from northeastern China. With a strict definition of VF, we found that the subgroup of high level LLV of above 400 copies $/ \mathrm{ml}$ and any level LLV coupled with blip of above 1000 copies/ml were high-risk LLV associated with VF. Moreover, we found that the duration of LLV also played an important role in contributing to the risk of VF. The association factors included high zenith VL, low nadir $\mathrm{CD} 4+\mathrm{T}$ cell counts, long term ART, Manchu, and subtype $\mathrm{B}^{\prime}$ infection.

The occurrence of LLV has not been reported in China, where uniform ART is provided freely; however, there is a lack of individual monitoring and treatment. In this study, we observed LLV from $38.7 \%$ of participants, and this was much higher than the LLV occurrence $(15-20 \%)$ in resource-rich settings with similar LLV definition criteria (50-1000 copies/ml) [18, 37]. Multiple potential factors could explain these differences, including different ART regimens and frequency of testing [6]. However, because of the strict definition of VF used in this study, the occurrence of VF was substantially lower than previous reported data on both resource-rich regions $[38,39]$ and resource-limited regions $[2,7]$. In a recent study from South Africa, 26\% VF according to standard of WHO (VL > 1000 copies $/ \mathrm{ml}$ once) showed spontaneous control of viral replication without changing treatment regimen, and therefore should not be considered as true VF [2]. In this study, we adjusted the standard of VF to two or more consecutive VL of above 1000 copies/ml with more than a 3-month interval. This new definition can avoid unnecessary ART regimen switches [2, 4], which is very important for resource-limited regions.

Using this new VF definition, we revealed that both level and duration of LLV were factors associated with VF. In this study, only LLV above 400 copies/ml could increase the risk of VF, which differed from several recent studies that implied a load of as low as 20 copies $/ \mathrm{ml}$ might lead to VF $[15,21]$. Such findings are usually obtained from source-rich regions, where abundant drugs are available and a completely different standard of VF (50 copies $/ \mathrm{ml}$ ) is used; therefore, this is not necessarily applicable in most resource-limited regions. Moreover, most previous studies have only evaluated the impact of different level LLV on VF, without considering the duration of LLV. A few studies found no significant association of low or medium levels LLV with varied duration [5, 6, 38]. However, the definition of VF in these studies was two consecutive viral loads of at least 500 copies $/ \mathrm{ml}$, which is rarely used in practice and differed from that used in this study.

Moreover, we also found that any level of LLV coupled with HLB increased the VF risk. In previous studies, HLB ( $>1000$ copies/ml) was usually defined as VF. However, in our study, 6.1\% (131/2155) participants (with average VL of 12,741 copies $/ \mathrm{ml}$ ) showed spontaneous control of viral replication in the follow up time. This phenomenon is more common in the cohort of South Africa (26\%), which we speculate is caused by irregular compliance or accident [2]. Previous reports indicated that MLLV is associated with increased occurrence of VF $[19,20]$; however, in the current study, we observed only an increasing trend of VF if lasting for 12 months. Thus, this potential association needs further validation.

Our current study implied that MLLV or LLLV were not associated with higher risk of VF, and therefore such cases should be only kept under observation without changing treatment. In contrast, a previous study suggested that patients with such level of LLV should be switched to other regimes or given stronger treatments immediately.

Some previous studies have considered both level and duration of LLV, which introduced a new parameter of viremia copy-years (VCY), combining the level and duration of virus replication, defined as the area under a patient's longitudinal VL curve. These studies found that the risk of VF rose with increasing VCY, which is consistent with the findings of the current study. However, no uniform threshold had been determined, because of the varied definitions of both LLV and VF [24, 25]. In 
contrast to previous studies, we focused only on the factors of high-risk LLV subgroup leading to VF, to identify conditions that need prompt treatment during ART. Although we used new definitions of LLV and VF, we found some similar risk factors as several resource-rich cohort studies, including the high zenith VL and low nadir CD4 + T cell counts [3, 22, 23]. In addition, we found that long-term ART, AZT + $3 \mathrm{TC}+\mathrm{NVP}$ or DDI + NRTI+NNRTI regimens, Manchu and subtype $\mathrm{B}^{\prime}$ infection were all risk factors in this study. In the early stage of this cohort, DDI-based regimen was used as first-line regimens according to the guide at that time, although these regimens were stopped several years later. It have been reported that DDI-based regimen-treated patients showed a higher cumulative risk of drug resistance [40] and the AZT + 3TC + NVP regimen had long-term sequelae and toxic and side effects [41, 42]; these findings offered a rational explanation for the increased frequency of high-risk LLV in this study.

According to this study, for patients with the baseline characters, such as high zenith VL, low nadir CD4 $+\mathrm{T}$ cell counts, Manchu, and subtype B', who were more likely to develop high-risk LLV, a more frequent follow-up should be recommended. The patients with LLV levels above 400 copies/ml, including both HLLV and HLB, and MLLV persisting longer than 12 months should be recommended to undergo drug resistance testing and blood drug concentration testing. On the other hand, for the patients with LLV levels below 200 copies $/ \mathrm{ml}$, follow-up can be continued.

Our study has some limitations. First, as a retrospective study, there may be some bias in participant selection. Second, $90 \%$ patients in this study self-reported good drug compliance, but the drug concentration in their plasma was not detected; thus, we were unable to assess the reliability of self-reported compliance. Third, about $5-10 \%$ of the social demographic and laboratory information were unavailable in this study, which might impact the results.

\section{Conclusion}

In conclusion, we observed the common occurrence of LLV among a local long-term ART cohort in China, among which, some LLV demonstrated with higher risk of VF and should be treated immediately. Our study also identified prognostic factors that can help determine the potential occurrence of high risk LLV. The results of this study provide a basis for development of differential treatment of LLV at various levels.

\section{Supplementary information}

Supplementary information accompanies this paper at https://doi.org/10. 1186/s12879-020-4837-y.

Additional file 1: Table S1. Baseline characteristics of the included participants $(N=2155)$.

\section{Abbreviations}

3TC: Lamivudine; ART: Antiretroviral therapy; AZT: Zidovudine; Blip: Viral blip; Cl: Confidence interval; D4T: Stavudine; DDI: Didanosine; DHHS: Department of Health and Human Services; EACS: European acquired immune deficiency syndrome Clinical Society; EFV: Efavirenz; HIV: Human immunodeficiency virus; HLB: High level blip; HLLV: High level LLV; HR: Hazard ratio;

IQR: Interquartile range; IR: Incidence rate; LLV: Low level viremia; NA: Not available; NNRTI: Non-nucleoside reverse transcriptase inhibitor; NRTIs: Nucleoside reverse transcriptase inhibitors; NVP: Nevirapine; pLLV: Persistent low level viremia; TDF: Tenofovir; VCY: Viremia copy-years; VF: Virologic failure; VL: Viral load; WHO: World Health Organization

\section{Acknowledgements}

We are grateful for the professor Hong Shang's contributions on the study design and manuscript writing. And we thank for the collaboration of the patients, medical and nursery staff and data mangers in this study. We would like to thank Editage (www.editage.cn) for English language editing. This study was funded by the Mega-projects of National Science Research for the 13th Five-Year Plan (2017ZX10201101), the Non-profit Central Research Institute Fund of Chinese Academy of Medical Sciences(2018PT31042), National Natural Science Foundation (81871637). None of the authors of this manuscript have conflict of interest.

\section{Authors' contributions}

$\mathrm{ZT}, \mathrm{DH}, \mathrm{WX}, \mathrm{TW}, \mathrm{ZB}$ collected the data used in this project. ZT, AM, HX designed the project. ZT analysed the data and wrote the first draft of a manuscript. ZT and HX then edited this first draft. All authors read, comment on and approved the final manuscript.

\section{Funding}

The Mega-projects of National Science Research for the 13th Five-Year Plan (2017ZX10201101), the Non-profit Central Research Institute Fund of Chinese Academy of Medical Sciences(2018PT31042) and National Natural Science Foundation (81871637) jointly funded the design of the study and collection, analysis, and interpretation of data and in writing the manuscript.

\section{Availability of data and materials}

The datasets used and/or analyzed during the current study are available from the corresponding author upon reasonable request.

Ethics approval and consent to participate

This study was approved by the ethics committee of the First Affiliated Hospital of China Medical University. In addition, written informed consents were obtained from all participants.

\section{Consent for publication \\ Not Applicable.}

\section{Competing interests}

The authors declare that they have no competing interests.

\section{Author details}

${ }^{1} \mathrm{NHC}$ Key Laboratory of AIDS Immunology (China Medical University), Department of Laboratory Medicine, The First Affiliated Hospital of China Medical University, No 155, Nanjing North Street, Heping District, Shenyang 110001, Liaoning Province, China. ${ }^{2}$ National Clinical Research Center for Laboratory Medicine, The First Affiliated Hospital of China Medical University, Shenyang 110001, China. ${ }^{3}$ Key Laboratory of AIDS Immunology of Liaoning Province, The First Affiliated Hospital of China Medical University, Shenyang 110001, China. ${ }^{4}$ Key Laboratory of AIDS Immunology, Chinese Academy of Medical Sciences, Shenyang 110001, China. ${ }^{5}$ Collaborative Innovation Center for Diagnosis and Treatment of Infectious Diseases, 79 Qingchun Street, Hangzhou 310003, China.

Received: 16 October 2019 Accepted: 30 January 2020

Published online: 17 February 2020

\section{References}

1. Paintsil E, Martin R, Goldenthal A, Bhandari S, Andiman W, Ghebremichael M. Frequent Episodes of Detectable Viremia in HIV Treatment-Experienced 
Children is Associated with a Decline in CD4+ T-cells Over Time. J AIDS Clin Res. 2016;7(4):565.

2. Hermans LE, Moorhouse M, Carmona S, et al. Effect of HIV-1 low-level viraemia during antiretroviral therapy on treatment outcomes in WHOguided south African treatment programmes: a multicentre cohort study. Lancet Infect Dis. 2018;18(2):188-97.

3. Bernal E, Gomez JM, Jarrin I, et al. Low-level Viremia is associated with clinical progression in HIV-infected patients receiving Antiretroviral treatment. J Acquir Immune Defic Syndr. 2018;78(3):329-37.

4. Pereira R, Ludwig DA, Mathew $S$, et al. Predicting viral failure in human immunodeficiency virus perinatally infected youth with persistent low-level Viremia on highly active Antiretroviral Therapy. J Pediatr Infect Dis Soc. 2019;8(4):303-9.

5. Sorstedt E, Nilsson S, Blaxhult A, et al. Viral blips during suppressive antiretroviral treatment are associated with high baseline HIV-1 RNA levels. BMC Infect Dis. 2016;16:305.

6. Joya C, Won SH, Schofield C, et al. Persistent low-level Viremia while on Antiretroviral Therapy is an independent risk factor for Virologic failure. Clin Infect Dis. 2019;69(12):2145-52.

7. Esber A, Polyak C, Kiweewa F, et al. Persistent low level viremia predicts subsequent virologic failure. Is it time to change the 3rd 90? Clin Infect Dis. 2019;69(5):805-12.

8. Dravid AN, Natrajan K, Kulkarni MM, et al. Discordant CSF/plasma HIV-1 RNA in individuals on virologically suppressive antiretroviral therapy in Western India. Medicine. 2018;97(8):e9969.

9. Consolidated guidelines on HIV prevention, diagnosis, treatment and care for key populations. World Health Organization. Available at: https://www. who.int/hiv/pub/guidelines/keypopulations-2016/en/9789241511124-eng. pdf. Accessed 1 July 2016.

10. Pernas B, Rego-Perez I, Tabernilla A, et al. Plasma mitochondrial DNA levels are inversely associated with HIV-RNA levels and directly with CD4 counts: potential role as a biomarker of HIV replication. J Antimicrob Chemother. 2017;72(11):3159-62.

11. Torres $B$, Guardo AC, Leal $L$, et al. Protease inhibitor monotherapy is associated with a higher level of monocyte activation, bacterial translocation and inflammation. J Int AIDS Soc. 2014;17(1):19246.

12. Swenson LC, Cobb B, Geretti AM, et al. Comparative performances of HIV-1 RNA load assays at low viral load levels: results of an international collaboration. J Clin Microbiol. 2014;52(2):517-23.

13. Panel on Antiretroviral Guidelines for Adults and Adolescents. Guidelines for the Use of Antiretroviral Agents in Adults and Adolescents Living with HIV. Department of Health and Human Services. Available at: http://www.aidsinfo. nih.gov/ContentFiles/AdultandAdolescentGL.pdf. Accessed 27 March 2018

14. Pernas B, Grandal M, Pertega S, et al. Any impact of blips and low-level viraemia episodes among HIV-infected patients with sustained virological suppression on ART? J Antimicrob Chemother. 2016;71(4):1051-5.

15. Maggiolo F, Callegaro A, Cologni G, et al. Ultrasensitive assessment of residual low-level HIV viremia in HAART-treated patients and risk of virological failure. J Acquir Immune Defic Syndr. 2012;60(5):473-82.

16. Ryom L, Boesecke C, Bracchi M, et al. Highlights of the 2017 European AIDS clinical society (EACS) guidelines for the treatment of adult HIV-positive persons version 9.0. HIV Med. 2018;19(5):309-15.

17. Zhang S, van Sighem A, Gras L, et al. Clinical significance of transient HIV type-1 viraemia and treatment interruptions during suppressive antiretroviral treatment. Antivir Ther. 2010;15(4):555-62.

18. Grennan JT, Loutfy MR, Su D, et al. Magnitude of virologic blips is associated with a higher risk for virologic rebound in HIV-infected individuals: a recurrent events analysis. J Infect Dis. 2012;205(8):1230-8.

19. Vandenhende MA, Perrier A, Bonnet F, et al. Risk of virological failure in HIV-1infected patients experiencing low-level viraemia under active antiretroviral therapy (ANRS C03 cohort study). Antivir Ther. 2015;20(6):655-60.

20. Laprise C, de Pokomandy A, Baril JG, Dufresne S, Trottier H. Virologic failure following persistent low-level viremia in a cohort of HIV-positive patients: results from 12 years of observation. Clin Infect Dis. 2013;57(10):1489-96.

21. Henrich TJ, Wood BR, Kuritzkes DR. Increased risk of virologic rebound in patients on antiviral therapy with a detectable HIV load $<48$ copies $/ \mathrm{mL}$. PLoS One. 2012;7(11):e50065.

22. Boillat-Blanco N, Darling KEA, Schoni-Affolter F, et al. Virological outcome and management of persistent low-level viraemia in HIV-1-infected patients: 11 years of the Swiss HIV Cohort study. Antivir Ther. 2014;20(2):165-75.

23. Farmer A, Wang X, Ganesan A, et al. Factors associated with HIV viral load "blips" and the relationship between self-reported adherence and efavirenz blood levels on blip occurrence: a case-control study. AIDS Res Ther. 2016;13:16.
24. Quiros-Roldan E, Raffetti E, Castelli F, et al. Low-level viraemia, measured as viraemia copy-years, as a prognostic factor for medium-long-term all-cause mortality: a MASTER cohort study. J Antimicrob Chemother. 2016;71(12):3519-27.

25. Wright ST, Hoy J, Mulhall B, et al. Determinants of viremia copy-years in people with HIV/AIDS after initiation of antiretroviral therapy. J Acquir Immune Defic Syndr. 2014;66(1):55-64.

26. AIDS and Hepatitis C Professional Group SolD, Chinese Medical Association, Chinese Center for Disease Control and Prevention. Chinese guidelines for diagnosis and treatment of HIV/AIDS (2018). Zhonghua Nei Ke Za Zhi. 2018:57(12):867-884.

27. Ruggiero A, Cozzi-Lepri A, Beloukas A, et al. Factors Associated With Persistence of Plasma HIV-1 RNA During Long-term Continuously Suppressive Firstline Antiretroviral Therapy. Open Forum Infect Dis. 2018;5(2):ofy032

28. Trickey A, May MT, Vehreschild JJ, et al. Survival of HIV-positive patients starting antiretroviral therapy between 1996 and 2013: a collaborative analysis of cohort studies. Lancet Hiv. 2017:4(8):E349-56.

29. Szubert AJ, Prendergast AJ, Spyer MJ, et al. Virological response and resistance among HIV-infected children receiving long-term antiretroviral therapy without virological monitoring in Uganda and Zimbabwe: observational analyses within the randomised ARROW trial. PLoS Med. 2017:14(11):e1002432.

30. Gunthard HF, Calvez V, Paredes R, et al. Human immunodeficiency virus drug resistance: 2018 recommendations of the international antiviral society-USA panel. Clin Infect Dis. 2019;68(2):177-87.

31. Zaccarelli M, Santoro MM, Armenia D, et al. Genotypic resistance test in proviral DNA can identify resistance mutations never detected in historical genotypic test in patients with low level or undetectable HIV-RNA. J Clin Virol. 2016;82:94-100.

32. Buchacz K, Lau B, Jing Y, et al. Incidence of AIDS-defining opportunistic infections in a multicohort analysis of HIV-infected persons in the United States and Canada, 2000-2010. J Infect Dis. 2016;214(6):862-72.

33. Torvinen-Kiiskinen S, Tolppanen AM, Koponen M, et al. Antidepressant use and risk of hip fractures among community-dwelling persons with and without Alzheimer's disease. Int J Geriatr Psychiatry. 2017;32(12):e107-15.

34. Hernandez-Ramirez RU, Qin L, Lin H, et al. Association of immunosuppression and HIV viremia with anal cancer risk in persons living with HIV in the United States and Canada. Clin Infect Dis. 2019. (Online ahead of print).

35. Palmer S, Maldarelli F, Wiegand A, et al. Low-level viremia persists for at least 7 years in patients on suppressive antiretroviral therapy. Proc Natl Acad Sci U S A. 2008;105(10):3879-84.

36. Mutoh $Y$, Nishijima T, Inaba $Y$, et al. Incomplete recovery of CD4 cell count, CD4 percentage, and CD4/CD8 ratio in patients with human immunodeficiency virus infection and suppressed Viremia during long-term Antiretroviral Therapy. Clin Infect Dis. 2018;67(6):927-33.

37. Elvstam $O$, Medstrand P, Yilmaz A, Isberg PE, Gisslen M, Bjorkman P. Virological failure and all-cause mortality in HIV-positive adults with lowlevel viremia during antiretroviral treatment. PLoS One. 2017;12(7):e0180761.

38. Antiretroviral Therapy Cohort C, Vandenhende MA, Ingle S, et al. Impact of low-level viremia on clinical and virological outcomes in treated HIV-1infected patients. Aids. 2015;29(3):373-83.

39. Teira R, Vidal F, Munoz-Sanchez P, et al. Very low level viraemia and risk of virological failure in treated HIV-1-infected patients. HIV Med. 2017;18(3): 196-203.

40. Di Mascio M, Markowitz M, Louie M, et al. Viral blip dynamics during highly active Antiretroviral Therapy. J Virol. 2003;77(22):12165-72.

41. LFD, SA D, MM L, et al. Evaluation of tolerance of zidovudine-lamivudinenevirapine combination in HIV 1 patients in Fann Teaching Hospital in Dakar. Bulletin de la Societe de pathologie exotique (1990). 2013;106(4):244-247.

42. Program NT. Toxicology and Carcinogenesis Studies of Mixtures of 3'-Azido3'-Deoxythymidine (AZT), Lamivudine (3TC), and Nevirapine (NVP) (CAS Nos. 30516-87-1, 134678-17-4, 129618-40-2) in Genetically Modified C3B6.129F1Trp53(tm1Brd) N12 Haploinsufficient Mice (in utero and postnatal gavage studies). National Toxicology Program genetically modified model report. 2013; undefined(16):1-236.

\section{Publisher's Note}

Springer Nature remains neutral with regard to jurisdictional claims in published maps and institutional affiliations. 